\title{
Research on obstacle avoidance technology of fixed wing formation based on improved artificial potential field method with stereo vision
}

\author{
Yu Liu*, and Xinhua Wang \\ Nanjing University of Aeronautics and Astronautics, Jiangsu Nanjing, China
}

\begin{abstract}
To solve the problem that distributed fixed wing formation cannot know all the other aircraft states through the ground station and may collide, the improved artificial potential field method based on binocular stereo vision was proposed. This method makes the fixed wing aircraft not need to obtain the position information of other fixed wing aircraft from the ground station, but only need the binocular stereo vision module to obtain the relative position and relative speed information of other fixed wing aircraft. These two parameters are used to improve the traditional artificial potential field method to meet the requirements of distributed fixed wing formation flight. The experimental results show that the improved artificial potential field method improves the safety and reliability of distributed fixed wing formation flying.
\end{abstract}

\section{Introduction}

With the development of artificial intelligence technology, intelligent UAV cluster cooperation to complete complex tasks has become a research hotspot. The traditional UAV cluster mainly adopts the centralized control method. A single UAV can obtain the information of other UAVs to avoid collision. However, when GPS information is abnormal or the central controller fails, it may lead to a collision accident in formation flight [1].

In order to solve the pain point of centralized control, distributed control is proposed as a UAV cluster control algorithm. In the distributed control method, a single UAV only needs to sense the status of other UAVs around it, and make self-adjustment according to other UAVs, without sharing information, making it more flexible [2]. So how to realize the environmental perception and obstacle avoidance of a single UAV has become an urgent problem to be solved.

At present, the main obstacle avoidance measurement methods are: ultrasonic measurement, 3D lidar survey, monocular vision detection and binocular vision detection. The feasibility of these methods is discussed respectively in the application scenario of obstacle avoidance of distributed fixed wing formation. First of all, the ultrasonic sensor

\footnotetext{
* Corresponding author: 243584914@qq.com
} 
can only provide two-dimensional single point information, and in the complex air flow conditions at high altitude, the acoustic wave is easy to be interfered, and the measurement accuracy is not high. 3D lidar can provide accurate 3D information of obstacles, but it is bulky and expensive, which increases the load of aircraft and is not suitable for engineering application. Secondly, monocular vision detection needs to estimate the distance according to the size of the target in the image, so the accuracy is not high [3]. And there are some problems, such as small field angle, small range of avoidable obstacle and so on. These common methods of obstacle avoidance are not suitable for distributed fixed wing formation. Therefore, in order to ensure the accuracy and meet the engineering application conditions, we choose to use binocular camera. It uses parallax to calculate distance, and its precision is higher than monocular camera. Moreover, the binocular camera is relatively small in size and moderate in price, which is suitable for UAV formation flying application scenarios.

In the selection of obstacle avoidance algorithm, artificial potential field method does not need global information and is suitable for distributed cluster control. At the same time, because of its simple structure, high calculation efficiency and convenience for the realtime control of the bottom layer, the artificial potential field method is selected in this paper [4-6]. However, due to the fast speed of fixed wing and the minimum turning radius constraint, the traditional artificial potential field method cannot meet the obstacle avoidance requirements and needs to be improved.

Based on the above analysis, this paper proposes an improved artificial potential field method which combines stereo vision with deep learning to realize the UAV's perception of the environment and obstacle avoidance. The second section introduces how to use binocular vision module to obtain the obstacle information, the third section introduces how to use the obstacle information to improve the artificial potential field method, and the fourth part has carried on the simulation verification test [7-8].

\section{Obstacle information extraction based on stereo vision}

By using binocular camera to recognize obstacles, the pixel coordinates of the ROI can be obtained. By combining the pixel coordinates with the $3 \mathrm{D}$ point cloud, the $3 \mathrm{D}$ space coordinates of the obstacle camera coordinate system can be obtained [10]. The specific implementation steps are as follows:

1. By using the Yolo detection algorithm, real-time detection is carried out on the picture taken by the left eye camera, and the pixel coordinates and types of obstacles in front of the UAV are obtained [9].

2. The area of the obstacle in the 3D point cloud is determined by combining the pixel coordinates of the ROI with the 3D dense point cloud information.

Filter the selected point cloud area. Filter out the matching error points and the points beyond the safe distance of the UAV.

3. K-means clustering is applied to the point cloud in the interest frame. Point cloud clusters with the widest distribution and the largest number are selected. Find the maximum and minimum values of the point clouds in the $\mathrm{x}$-axis and $\mathrm{Y}$-axis directions. Find the maximum value of the point cloud cluster in the $\mathrm{z}$-axis direction as the distance from the obstacle, and set it as $\mathrm{Z}$. The center point of the contour is taken as the position of the obstacle in the camera coordinate system. The coordinates of the obstacle relative to the UAV are:

$$
\left(X_{p o}, Y_{p o}, Z_{p o}\right)=\left(\frac{X_{\max }-X_{\min }}{2}, \frac{Y_{\max }-Y_{\min }}{2}, Z\right)
$$


4. The relative velocity vector can be calculated by comparing the relative positions of obstacles in the front and back frames $v_{p o}$.

\section{Obstacle avoidance algorithm of APF}

\subsection{Traditional artificial potential field method}

Khatib proposed the artificial potential field method based on virtual force method in 1986 , which is a local obstacle avoidance algorithm [10]. The force field of UAV is as follows:

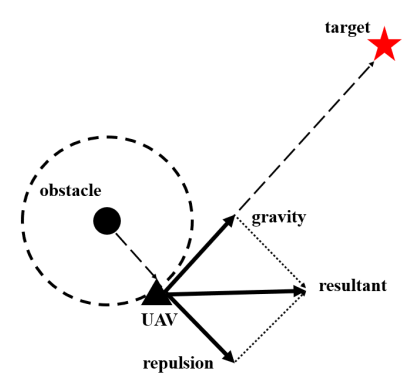

Fig. 1. The force of UAV in the field of influence.

$$
\begin{gathered}
T_{\mathrm{s}}(l, t)=T_{\mathrm{g}}(l, t) \\
T_{\mathrm{s}}(l, t)=T_{\mathrm{g}}(l, t) T_{\mathrm{b}}(x \rightarrow-\beta, t)=0
\end{gathered}
$$

Suppose that the position of fixed wing aircraft in space is $p$, the position of target point is $q$, and the force field function is as follows:

1. Gravitational force field function

The gravitational field function and gravity of the aircraft are as follows:

$$
\begin{gathered}
U_{a t t}(p)=\frac{1}{2} k_{a} D_{p t}^{2}(p, t) \\
F_{a t t}(p)=-\nabla U_{a t t}(p)=-k_{a} D_{p t}(p, t)
\end{gathered}
$$

$k_{a}$ is the gain coefficient of the gravitational potential energy field, and $D_{p t}(p, t)$ is the Euclidean distance from the point $p$ of the UAV to the point $\mathrm{t}$ of the target.

2. Repulsive force field function

The repulsive field function and repulsion of the aircraft are as follows:

$$
U_{r e p}(p)=\left\{\begin{array}{cr}
\frac{1}{2} k_{r}\left(\frac{1}{D_{p o i}\left(p, o_{i}\right)}-\frac{1}{\rho_{0}}\right)^{2}, D_{p o i}\left(p, o_{i}\right) \leq \rho_{0} \\
0 \quad, D_{p o i}\left(p, o_{i}\right)>\rho_{0}
\end{array}\right.
$$




$$
F_{r e p}(p)=-\nabla U_{r e p}(p)=\left\{\begin{array}{rr}
k_{r}\left(\frac{1}{D_{p o i}\left(p, o_{i}\right)}-\frac{1}{\rho_{0}}\right) \frac{1}{D_{p o i}^{2}\left(p, o_{i}\right)} \nabla D_{p o i}\left(p, o_{i}\right), D_{p o i}\left(p, o_{i}\right) \leq \rho_{0} \\
0 & , D_{p o i}\left(p, o_{i}\right)>\rho_{0}
\end{array}\right.
$$

$k_{r}$ is the gain coefficient of the repulsive potential energy field, and $D_{p o i}\left(p, o_{i}\right)$ is the distance between the UAV $p$ and the $i$ obstacle.

Artificial potential field method does not need global information and is suitable for distributed cluster control. But it also has the following disadvantages:

1. Unable to reach target when the gravity is small and the repulsion is large.

2. Fall into local optimum when repulsion and gravitation are equal, and the direction is opposite, which may lead to the continuous positive vibration.

3. The traditional artificial potential field method only considers the distance factor, but the fixed wing needs to increase the speed factor because of the restriction of its high speed and minimum turning radius, so the traditional method needs to be improved.

\subsection{Improved artificial potential field method}

For the obstacle avoidance problem of fixed wing formation, it is not easy to theorize due to too many factors that need to be considered in practice. Therefore, the following reasonable assumptions are made:

1. It is assumed that the fixed wing formation is always flying at the same altitude. Because there is no collision problem in different altitude flight, it is not discussed in this paper.

2. Obstacles are divided into static obstacles and dynamic obstacles. Static obstacles include no fly zones, buildings, etc. the location of such obstacles is known. Moving obstacles are other aircraft in the formation.

3. The relative position and speed of moving obstacles relative to the machine are measured by binocular stereo vision module.

\subsubsection{Improved gravitational force field function}

$$
\begin{gathered}
U_{a t t}\left(p, v_{p}\right)=\xi_{p} D_{p t}^{m}(p, t)+\xi_{v} v_{p}^{n} \\
F_{p a t t}(p)=m \xi_{p} D_{p t}^{m-1}(p, t) X_{p r g} \\
F_{v a t t}(v)=n \xi_{v} v_{p}^{n-1}
\end{gathered}
$$

$X_{p r g}$ is the unit vector of the position of the target point relative to the UAV $p$.

\subsubsection{Improved repulsive force field function}

The relative velocity of obstacles and UAVs is introduced into the repulsive force field function according to the direct flight characteristics of fixed wing aircraft and the minimum turning radius constraint. The component $v_{p o z}$ of the relative velocity $v_{p o}$ in the $\mathrm{z}$-axis direction is used as the judgment basis. Only when $v_{p o z}<0$, the effect of relative 
velocity is considered. Because when $v_{p o z}>0$, UAV $p$ will not hit the obstacle in front. The improved repulsive force field function is as follows:

$$
U_{r e p}\left(p, v_{p o}\right)=\left\{\begin{array}{c}
\frac{1}{2} \xi_{1}\left(\frac{1}{D_{p o i}\left(p, o_{i}\right)}-\frac{1}{\rho_{0}}\right)^{2}, D_{p o i}\left(p, o_{i}\right) \leq \rho_{0} \text { and } \quad v_{p o z} \geq 0 \\
\frac{1}{2} \xi_{1}\left(\frac{1}{D_{p o i}\left(p, o_{i}\right)}-\frac{1}{\rho_{0}}\right)^{2}+\xi_{2} v_{p o}^{2}, D_{p o i}\left(p, o_{i}\right) \leq \rho_{0} \text { and } \quad v_{p o z}<0 \\
0 \quad, D_{p o i}\left(p, o_{i}\right)>\rho_{0}
\end{array}\right.
$$

where, $\xi_{1}, \xi_{2}$ is the repulsion coefficient and $\rho_{0}$ is the influence range of obstacles.

$$
F_{r e p}(p)=-\nabla U_{r e p}\left(p, v_{p o}\right)=F_{\text {prep }}(p)+F_{\text {vrep }}\left(v_{p o}\right)
$$

Among them:

$$
F_{r e p}\left(p, v_{p o}\right)=\left\{\begin{array}{c}
\xi_{1}\left(\frac{1}{D_{p o i}\left(p, o_{i}\right)}-\frac{1}{\rho_{0}}\right) \frac{1}{D_{p o i}^{2}\left(p, o_{i}\right)} X_{p r o}, D_{p o i}\left(p, o_{i}\right) \leq \rho_{0} \text { and } \quad v_{p o z} \geq 0 \\
\xi_{1}\left(\frac{1}{D_{p o i}\left(p, o_{i}\right)}-\frac{1}{\rho_{0}}\right) \frac{1}{D_{p o i}^{2}\left(p, o_{i}\right)} X_{p r o}+2 \xi_{2}\left\|v_{p o}\right\|, D_{p o i}\left(p, o_{i}\right) \leq \rho_{0} \text { and } \quad v_{p o z}<0 \\
0 \quad, D_{p o i}\left(p, o_{i}\right)>\rho_{0}
\end{array}\right.
$$

$X_{p r o}$ is the unit vector of the position of UAV $p$ relative to obstacle $O$.If the repulsion force provided by the speed parameter is increased, the buffer time is provided, so that the fixed wing can move away slowly, avoiding large angle maneuver. This improvement meets the requirements of fixed wing flight, and can solve the problems of local optimum and target unreachable.

\subsection{Simulation results and analysis}

Simulation is used to verify the improved artificial potential field method. The simulation environment is Matlab 2017A under Windows 10 operating system. Take two fixed wing aircraft as an example, in which fixed wing aircraft $\mathrm{A}$ starts from point $\mathrm{S}$ to point $\mathrm{E}$, and on the way, it needs to avoid two static obstacles and another fixed wing aircraft B flying in a straight line. The purpose of simulation is to verify that the improved method can meet the flight requirements of fixed wing formation by comparing the former method with the latter one. 


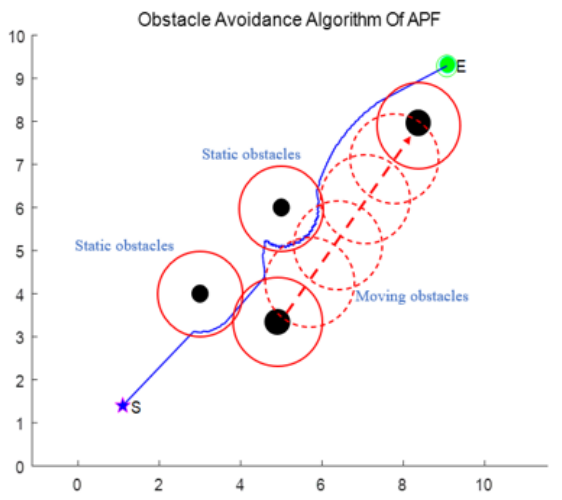

Fig. 2. Traditional artificial potential field method to avoid obstacles.

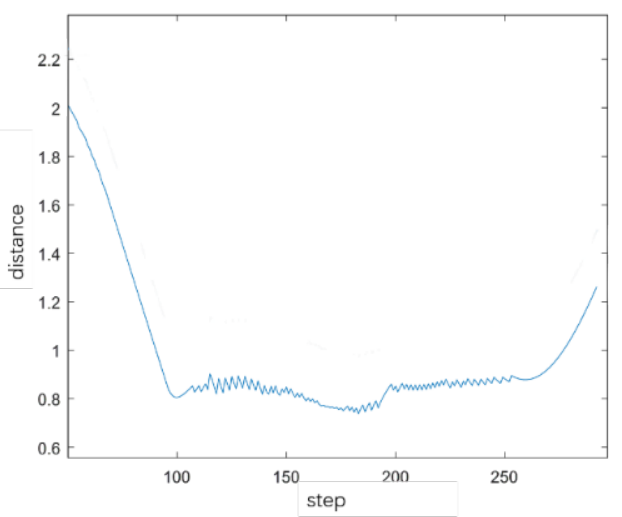

Fig. 3. Distance between fixed wings A and B.

As shown in the Fig.2, when fixed wing A passes through the second static obstacle, there is a movement track of large maneuver turning, which does not conform to the flight characteristics of fixed wing aircraft. At the same time, the distance curve in Fig. 3 shows high frequency oscillation, which indicates that the speed of aircraft A changes rapidly, not only increases energy consumption but also is not conducive to flight safety. In order to solve these problems, the artificial potential field method is improved, and the velocity variable is introduced into the force field function. The simulation diagram is as follows.

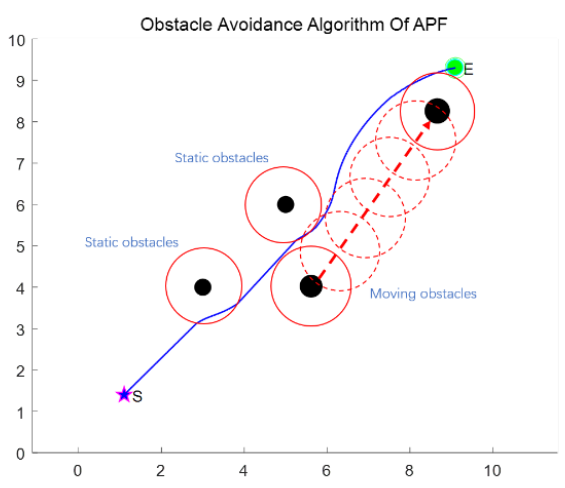

Fig. 4. Improved artificial potential field method to avoid obstacles. 


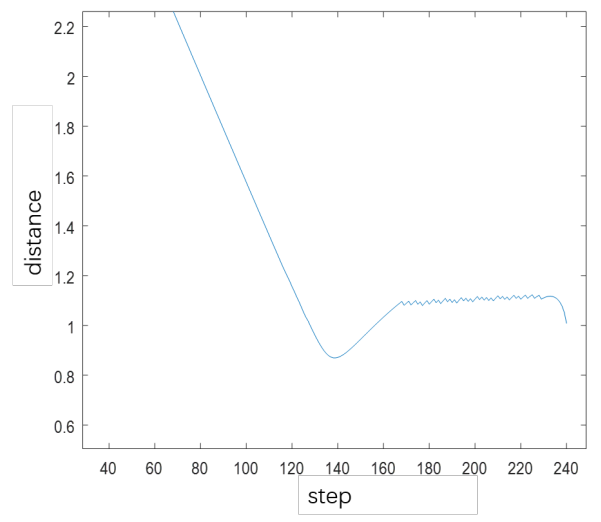

Fig. 5. Distance between fixed wings A and B.

As shown in the Fig. 4, the improved artificial potential field method can ensure the safety of the fixed wing aircraft without changing the course in large maneuver. From Fig. 5 , it can be seen that fixed wing A enters into the influence range of B at high speed. Due to the consideration of the influence of speed, it will suffer a lot of repulsion force, so that it quickly stays out of the influence range. At the same time, the speed of fixed wing A will not fluctuate at high frequency, which will reduce the impact on the performance of fixed wing a and reduce the energy consumption.

\section{Conclusion}

In this paper, for the application of distributed fixed wing formation, binocular stereo vision is used to obtain the relative position information and relative speed information of other aircraft around, and the relative speed is used as a parameter to improve the force field function. The experimental results show that the improved artificial potential field method can meet the flight characteristics of the fixed wing and prevent the aircraft from turning at a large angle. At the same time, the addition of velocity component ensures the safety of flight and reduces the high frequency oscillation.

\section{References}

1. Rostami S M H, Sangaiah A K, Wang J, et al. Obstacle avoidance of mobile robots using modified artificial potential field algorithm[J]. EURASIP Journal on Wireless Communications and Networking, 2019, 2019(1): 70.

2. Zhang M, Liu H H T. Cooperative tracking a moving target using multiple fixed-wing UAVs[J]. Journal of Intelligent \& Robotic Systems, 2016, 81(3-4): 505-529.

3. Jia Z, Yang J, Liu W, et al. Improved camera calibration method based on perpendicularity compensation for binocular stereo vision measurement system[J]. Optics express, 2015, 23(12): 15205-15223.

4. Cao Z L, Yan Z H, Wang H. Summary of binocular stereo vision matching technology[J]. Journal of Chongqing University of Technology (Natural Science), 2015, 29(2): 70-75.

5. Simony M, Milzy S, Amendey K, et al. Complex-yolo: An euler-region-proposal for real-time $3 \mathrm{~d}$ object detection on point clouds[C]//Proceedings of the European Conference on Computer Vision (ECCV). 2018: 0-0. 
6. Laroca R, Severo E, Zanlorensi L A, et al. A robust real-time automatic license plate recognition based on the YOLO detector[C]//2018 International Joint Conference on Neural Networks (IJCNN). IEEE, 2018: 1-10.

7. Tijmons S, de Croon G C H E, Remes B D W, et al. Obstacle avoidance strategy using onboard stereo vision on a flapping wing MAV[J]. IEEE Transactions on Robotics, 2017, 33(4): 858-874.

8. Peng Y, Qu D, Zhong $\mathrm{Y}$, et al. The obstacle detection and obstacle avoidance algorithm based on 2-d lidar[C]//2015 IEEE international conference on information and automation. IEEE, 2015: 1648-1653.

9. Varga M, Basiri M, Heitz G, et al. Distributed formation control of fixed wing micro aerial vehicles for area coverage[C]//2015 IEEE/RSJ International Conference on Intelligent Robots and Systems (IROS). IEEE, 2015: 669-674.

10. Oleynikova H, Honegger D, Pollefeys M. Reactive avoidance using embedded stereo vision for mav flight[C]//2015 IEEE International Conference on Robotics and Automation (ICRA). IEEE, 2015: 50-56. 